\title{
TENSOR PRODUCTS OF IDEMPOTENT SEMIMODULES. AN ALGEBRAIC APPROACH
}

\author{
G. L. Litvinov, V. P. Maslov, and G. B. Shpiz
}

\begin{abstract}
We study idempotent analogs of topological tensor products in the sense of A. Grothendieck. The basic concepts and results are simulated on the algebraic level. This is one of a series of papers on idempotent functional analysis.
\end{abstract}

KEY WORDS: idempotent functional analysis, idempotent semiring, idempotent semimodule, tensor product, polylinear mapping, nuclear operator.

Dedicated to S.G. Krein on the occasion of his 80th birthday

\section{INTRODUCTION}

We construct and study tensor products in some natural categories of idempotent semimodules. In idempotent analysis, these tensor products play a role similar to that of the topological tensor products constructed by A. Grothendieck [1] in functional analysis. However, we point out that the idempotent versions of the basic notions and results are fairly different from their conventional analogs.

The basic concepts and results (including those of "topological" nature) are simulated on the algebraic level; the point is that the operation of idempotent addition can be defined for infinitely many summands. The present paper is one of a series of papers on idempotent functional analysis, which is an abstract version of idempotent analysis in the sense of [2-9]. In the subsequent publications, we intend to study the links between idempotent tensor products, idempotent linear integral and nuclear operators, and traces of operators.

\section{$\S 1$. BASIC CONCEPTS}

1.1. We recall that an idempotent semigroup is an additive semigroup $S$ with commutative addition $\oplus$ such that $x \oplus x=x$ for all elements $x \in S$. If $S$ has a neutral element, then this element is denoted by $\mathbf{0}$. Any idempotent semigroup is a partially ordered set with respect to the standard order defined as follows: $x \preccurlyeq y$ if and only if $x \oplus y=y$. This is obviously well defined, and $x \oplus y=\sup \{x, y\}$. Thus each idempotent semigroup is an upper semilattice, and moreover, the notions of an idempotent semigroup and an upper semilattice essentially coincide [10].

The definitions given below are partly borrowed from [11, 12]. An idempotent semigroup $S$ is said to be a-complete (or algebraically complete) if it is complete

Appears in: Math. Notes 64:4 (1999) 479-489, as the English translation of the paper published in Mat. Zametki 64:4 (1999) 572-585. 
as an ordered set, i.e., each subset $X \subset S$ has the least upper bound $\sup X$, denoted also by $\oplus X$, and the greatest lower bound inf $X$, denoted also by $\wedge X$. This semigroup is said to be b-complete (or boundedly complete) if each subset $X \subset S$ (possibly, empty) that is bounded above has the least upper bound $\oplus X$ (in this case each nonempty subset $Y \subset S$ has the greatest lower bound $\wedge Y$, and $S$ is a lattice). Note that each $a$ - or $b$-complete idempotent semigroup has a zero $\mathbf{0}$, which coincides with $\oplus \varnothing$, where $\varnothing$ is the empty set. Needless to say, $a$-completeness implies $b$-completeness. The cut completion procedure [10] provides an embedding $S \rightarrow \widehat{S}$ of an arbitrary idempotent semigroup $S$ in an $a$-complete idempotent semigroup $\widehat{S}$ (called the normal completion of $S$ ); furthermore, $\widehat{\widehat{S}}=S$. In a similar way, one defines the $b$-completion $S \rightarrow \widehat{S}_{b}$ : if $S \ni \infty=\sup S$, then $\widehat{S}_{b}=S$; otherwise, $\widehat{S}=\widehat{S}_{b} \cup\{\infty\}$. An arbitrary $b$-complete idempotent semigroup $S$ also can differ from $\widehat{S}$ only by the element $\infty=\sup S$.

Let $S$ and $T$ be $b$-complete idempotent semigroups. A homomorphism $f: S \rightarrow T$ will be called a b-homomorphism if $f(\oplus X)=\oplus f(X)$ for each bounded subset $X$ of $S$. If a $b$-homomorphism $f$ extends to be a homomorphism $\widehat{S} \rightarrow \widehat{T}$ of the normal completions, and moreover, if $f(\oplus X)=\oplus f(X)$ for all $X \subset S$, then $f$ will be called an a-homomorphism. Each $a$-homomorphism is a $b$-homomorphism. If the semigroup $S$ is $a$-complete, then each $b$-homomorphism is an $a$-homomorphism. If $S$ and $T$ are topological idempotent semigroups, then a homomorphism $f: T \rightarrow S$ that takes zero to zero is an $a$-homomorphism if and only if $f$ is lower semicontinuous $[11,12]$.

1.2. An idempotent semiring (for brevity, we sometimes simply say "semiring") is an idempotent semigroup $(K, \oplus)$ equipped with an associative multiplication $\odot$ such that both distributivity conditions hold. If the multiplication is commutative, then the idempotent semiring is said to be commutative. An element $\mathbf{1} \in K$ is called the unit of the semirings $K$ if $x \odot \mathbf{1}=\mathbf{1} \odot x=x$ for all $x \in K$. An element $\mathbf{0} \in K, \mathbf{0} \neq \mathbf{1}$, is called the zero of the semirings $K$ if $x \oplus \mathbf{0}=x$ and $x \odot \mathbf{0}=$ $\mathbf{0} \odot x=\mathbf{0}$ for all $x \in K$. In this paper we consider only idempotent semirings with unit. The presence of zero is usually also assumed (unless specified otherwise). An idempotent semifield (or simply semifield) is a commutative idempotent semiring in which every nonzero element is invertible with respect to multiplication. An idempotent semiring $K$ is said to be $a$-complete (respectively, $b$-complete) if $K$ is an $a$-complete (respectively, $b$-complete) idempotent semigroup and if the generalized distributivity laws $k \odot(\oplus X)=\oplus(k \odot X)$ and $(\oplus X) \odot k=\oplus(X \odot k)$ hold for each subset (respectively, each bounded subset) $X \subset K$ and each $k \in K$. It follows from the generalized distributivity that each $a$-complete or $b$-complete idempotent semiring has a zero element, which coincides with $\oplus \varnothing$. The notion of an $a$-complete idempotent semiring coincides with that of a complete dioid in the sense of $[13,14]$.

The set $\mathbb{R}(\max ,+)$ of real numbers equipped with the idempotent addition $\oplus=$ max and the multiplication $\odot=+$ is an example of an idempotent semiring; in this case $\mathbf{1}=0$. By supplementing this idempotent semiring with the element $\mathbf{0}=-\infty$, we obtain the $b$-complete semiring $\mathbb{R}_{\max }=\mathbb{R} \cup\{-\infty\}$ with the same operations and with zero. By supplementing $\mathbb{R}_{\max }$ with the element $+\infty$ and by setting $\mathbf{0} \odot(+\infty)=\mathbf{0}, x \odot(+\infty)=+\infty$ for $x \neq \mathbf{0}, x \oplus(+\infty)=+\infty$ for all $x$, we obtain the $a$-complete idempotent semiring $\widehat{\mathbb{R}}_{\max }=\mathbb{R}_{\max } \cup\{+\infty\}$. The standard order on $\mathbb{R}(\max ,+), \mathbb{R}_{\max }$, and $\widehat{\mathbb{R}}_{\max }$ coincides with the usual order. The 
idempotent semirings $\mathbb{R}(\max ,+), \mathbb{R}_{\max }$ are semifields. On the other hand, an $a$ complete idempotent semiring other than $\{\mathbf{0}, \mathbf{1}\}$ cannot be a semifield. There is an important class of examples related to (topological) vector lattices (e.g., see [15, Chap. V] and [10]). By defining the sum $x \oplus y$ as $\sup \{x, y\}$ and the multiplication $\odot$ as addition of vectors, one can interpret vector lattices as idempotent semifields. By supplementing a complete vector lattice (in the sense of $[10,15]$ ) with the element 0, we obtain a $b$-complete semifield. Next, we can add the "infinite" element, thus obtaining an $a$-complete idempotent semiring (coinciding as the ordered set with the normal completion of the original lattice).

1.3. Let $V$ be an idempotent semigroup and $K$ an idempotent semiring. Suppose that a multiplication $k, x \mapsto k \odot x$ of elements of $V$ by elements of $K$ is given, and moreover, this multiplication is associative, distributes over the addition in $V$, and satisfies $1 \odot x=x$ for all $x \in V$. In this case the semigroup $V$ is called an idempotent semimodule (or simply semimodule) over $K$. An element $\mathbf{0}_{V} \in V$ is called the zero of the semimodule $V$ if $k \odot \mathbf{0}_{V}=\mathbf{0}_{V}, \mathbf{0}_{K} \odot x=\mathbf{0}_{V}$, and $\mathbf{0}_{V} \oplus x=x$ for all $k \in K$ and $x \in V$. Let $V$ be a semimodule over a $b$-complete idempotent semiring $K$. This semimodule is said to be $b$-complete if it is $b$-complete as an idempotent semigroup and if the generalized distributivity laws $(\oplus Q) \odot x=\oplus(Q \odot x)$ and $k \odot(\oplus X)=\oplus(k \odot X), k \in K, x \in X$, hold for any bounded subsets $Q \subset K$ and $X \subset V$. This semimodule is said to be $a$-complete if it is $b$-complete and contains the element $\infty=\sup V$.

Let $V$ and $W$ be idempotent semimodules over an idempotent semiring $K$. A mapping $p: V \rightarrow W$ is said to be linear (over $K$ ) if $p(x \oplus y)=p(x) \oplus p(y)$ and $p(k \odot x)=k \odot p(x)$ for all $x, y \in V$ and $k \in K$. Let the semimodules $V$ and $W$ be $b$-complete. A linear mapping $p: V \rightarrow W$ is said to be $b$-linear if it is a $b$-homomorphism of idempotent semigroups and a-linear if it extends to be an $a$ homomorphism of the normal completions $\widehat{V}$ and $\widehat{W}$. Every $a$-linear mapping is $b$-linear. If a mapping $V \rightarrow W$ is $b$-linear and the semimodule $V$ is $a$-complete, then this mapping is $a$-linear. The $a$-linearity is similar to continuity (semicontinuity) for linear mappings. A semiring $K$ or the normal completion $\widehat{K}$ of a semifield $K$ is a semimodule over $K$. If $W$ coincides with $K$ (or $\widehat{K}$ ), then the linear mapping $p$ is called a linear functional. Linear (respectively, $b$-linear) mappings will also be referred to as linear (respectively, b-linear) operators.

In analysis, the most important examples of idempotent semimodules and spaces are either subsemimodules of topological vector lattices [15] (possibly coinciding with these) or their duals (that is, semimodules of linear functionals with some regularity condition, say, of $a$-linear functionals).

Remark 1. Clearly, idempotent semimodules over a given idempotent semiring form a category with linear mappings as morphisms. In the present paper we mainly deal with another category, which is formed by $b$-complete idempotent semimodules over a given $b$-complete idempotent semiring with $b$-linear mappings as morphisms, and also with the full subcategory of $a$-complete semimodules with $a$-linear mappings as morphisms. Note that these categories are not additive (it is natural to say that they are semiadditive); for the basic notions of the category theory, e.g., see [16].

In what follows, unless specified otherwise, we consider only $b$-complete (including $a$-complete) idempotent semirings and idempotent semimodules. 


\section{§2. Direct Products and Sums of $b$-Complete Idempotent Semimodules}

Let $K$ be a $b$-complete idempotent semiring, and let $\left\{V_{\alpha}\right\}_{\alpha \in A}$ be a family of $b$-complete idempotent semimodules over $K$. We represent an element of the direct product of all sets of this family as a (generally speaking, infinite) sequence ("vector") $x=\left\{x_{\alpha}\right\}=\left(\ldots, x_{\alpha}, \ldots\right)$, where $x_{\alpha} \in V_{\alpha}$. The componentwise operations $x \oplus y=\left\{x_{\alpha}+y_{\alpha}\right\}=\left(\ldots, x_{\alpha} \oplus y_{\alpha}, \ldots\right)$ and $k \odot x=\left\{k \odot x_{\alpha}\right\}=\left(\ldots, k \odot x_{\alpha}, \ldots\right)$, $k \in K$, make the direct product of the sets $V_{\alpha}, \alpha \in A$, an idempotent semimodule, which is called the direct product of the semimodules $V_{\alpha}, \alpha \in A$, and is denoted by $\prod_{\alpha} V_{\alpha}$. The direct product of semimodules $V_{1}, \ldots, V_{n}$ will also be denoted by $V_{1} \times \cdots \times V_{n}$. For each index $\alpha \in A$, we define a projection $p_{\alpha}: \prod_{\alpha} V_{\alpha} \rightarrow V_{\alpha}$ by setting $p_{\alpha}(x)=x_{\alpha}$ and an embedding $i_{\alpha}: V_{\alpha} \rightarrow \prod_{\alpha} V_{\alpha}$ by setting $(i(x))_{\alpha}=x$ and $(i(x))_{\beta}=\mathbf{0}$ for $\alpha \neq \beta$.

Proposition 1. The semimodule $\prod_{\alpha} V_{\alpha}$ is a-complete (respectively, b-complete) if so are all semimodules $V_{\alpha}$.

This assertion readily follows from the definitions.

Proposition 2. Let $\left\{V_{\alpha}\right\}_{\alpha \in A}$ be a family of b-complete semimodules over $K$, and let $V$ be a b-complete semimodule over $K$. Then the following assertions hold:

1) for each family of b-linear mappings $f_{\alpha}: V \rightarrow V_{\alpha}$ there exists a unique b-linear mapping $f: V \rightarrow \prod_{\alpha} V_{\alpha}$ such that $p_{\alpha} f=f_{\alpha}$ for each index $\alpha \in A$;

2 ) if the indexing set $A$ is finite, then for each family of b-linear mappings $f_{\alpha}: V_{\alpha} \rightarrow V$ there exists a unique b-linear mapping $f: \prod_{\alpha} V_{\alpha} \rightarrow V$ such that $f i_{\alpha}=f_{\alpha}$ for each index $\alpha \in A$;

3) if all semimodules $V$ and $V_{\alpha}, \alpha \in A$, are a-complete, then for any indexing set $A$ and any family of a-linear mappings $f_{\alpha}: V_{\alpha} \rightarrow V$ there exists a unique a-linear mapping $f: \prod_{\alpha} V_{\alpha} \rightarrow V$ such that $f i_{\alpha}=f_{\alpha}$ for each $\alpha \in A$.

Proof. We start from assertion 1). We set $f(x)=\left\{f_{\alpha}(x)\right\}$. One can readily see that a subset of $\prod_{\alpha} V_{\alpha}$ is bounded if and only if the projections of this subset on all components are bounded. This, together with the definitions, readily implies that $f$ is $b$-linear; moreover, by construction, $p_{\alpha} f=f_{\alpha}$ for all $\alpha \in A$. The uniqueness of the desired mapping is also obvious, and the proof of assertion 1) is complete. The mapping $f$ is called the direct product of the mappings $f_{\alpha}$.

To prove assertion 2), we set $f\left(\left\{x_{\alpha}\right\}\right)=\bigoplus_{\alpha} f_{\alpha}\left(x_{\alpha}\right)$. One can readily see that if $X \subset \prod_{\alpha} V_{\alpha}$ is a bounded subset, then so is the set $\left\{f_{\alpha}\left(x_{\alpha}\right) \mid \alpha \in A, x_{\alpha} \in p_{\alpha}(X)\right\}$. It follows that the mapping $f$ is well defined; the relations $f i_{\alpha}=f_{\alpha}$ for all $\alpha \in A$ and the fact that $f$ is $b$-linear and unique can be verified directly. This mapping is called the direct sum of the mappings $f_{\alpha}$.

The proof of assertion 3) is similar. The finiteness of the indexing set was used only in the proof of the boundedness of the set $\left\{f_{\alpha}\left(x_{\alpha}\right) \mid \alpha \in A, x_{\alpha} \in p_{\alpha}(X)\right\}$. However, for an $a$-complete semimodule $V$ this holds automatically. It remains to notice that under the assumptions of this assertion $a$-linearity coincides with $b$-linearity.

Thus the proof of Proposition 2 is complete.

Remark 2. In the language of the category theory, Proposition 2 has the following meaning. In the category of $b$-complete idempotent semimodules there exists a 
(categorical) direct product of an arbitrary family of semimodules $V_{\alpha}$; this product coincides with $\prod_{\alpha} V_{\alpha}$. If the family is finite, then there also exists a (categorical) direct sum $\sum_{\alpha} V_{\alpha}$ semimodules $V_{\alpha}$, which coincides with the direct sum $\prod_{\alpha} V_{\alpha}$. In the category of $a$-complete semimodules, for an arbitrary family of semimodules $V_{\alpha}$ there exist a (categorical) direct sum $\sum_{\alpha} V_{\alpha}$ and a direct product $\prod_{\alpha} V_{\alpha}$, which coincide with each other and with the direct product in the category of $b$-complete semimodules.

\section{$\S 3$. Tensor Products of $b$-Complete Semimodules}

Let $\left\{V_{\alpha}\right\}_{\alpha \in A}$ be a family of $b$-complete idempotent semimodules over a given $b$-complete commutative idempotent semiring $K$, and let $V=\prod_{\alpha} V_{\alpha}$.

By $T$ we denote the set of formal sums of the form

$$
t=\bigoplus_{x=\left\{x_{\alpha}\right\} \in X} \lambda(x) \odot \bigotimes_{\alpha} x_{\alpha},
$$

where $X \subset V, x=\left\{x_{\alpha}\right\}=\left(\ldots, x_{\alpha}, \ldots\right)$, and $\lambda$ is an arbitrary mapping of $X$ into $K$. An element of the form (1) will be called a representation of a tensor (in what follows we define a tensor as a class of equivalent representations). The natural (formal) idempotent addition (which should not be confused with the addition in $V=\prod_{\alpha} V_{\alpha}$ ) and the multiplication by elements $k \in K$ (which takes each function $\lambda(x)$ to $x \mapsto k \odot \lambda(x))$ make $T$ a semimodule over $K$.

We say that a representation of the form (1) is bounded if $X$ is bounded in $V$ and $\lambda(x)$ is a bounded function on $X$ (ranging in $K$ ). The set of all bounded representations will be denoted by $T_{b}$. Clearly, $T_{b}$ is a subsemimodule of $T$. One can readily see that the semimodule $T_{b}$ is $b$-complete.

As usual, we equip $T$ and $T_{b}$ with the equivalence relation generated by the identities

$$
\begin{aligned}
& k \odot\left(\cdots \otimes x_{\alpha} \otimes \cdots\right)=\left(\cdots \otimes k \odot x_{\alpha} \otimes \cdots\right), \\
& \left(\cdots \otimes\left(\oplus X_{\alpha}\right) \otimes \cdots\right)=\bigoplus_{x \in X_{\alpha}}(\cdots \otimes x \otimes \cdots),
\end{aligned}
$$

where $k \in K, X_{\alpha} \subset V_{\alpha}$, some value of the index $\alpha$ is chosen, and all respective components (formal factors) on the right- and left-hand sides in (2) and (3) coincide except for those written out explicitly. Relation (2) permits one to replace the sum (1) by an equivalent representation of the form

$$
t=\bigoplus_{x=\left\{x_{\alpha}\right\} \in X} \bigotimes_{\alpha} x_{\alpha} .
$$

In what follows we deal with representations of the form (4); in this case, by some abuse of speech, the set $X \subset \prod_{\alpha} V_{\alpha}$ occurring in (4) will also be called a representation of a given tensor. The addition of tensors of the form (4) corresponds to the union of their representations (summands). This addition must be idempotent; hence, by summing a given representation with all equivalent representations, we obtain the same tensor (up to equivalence). The corresponding representation is naturally called complete; we identify a tensor, i.e., a class of equivalent representations, with the corresponding complete representation. We shall assume that the complete representation always contains the element $\mathbf{0}_{V}=\bigotimes_{\alpha} \mathbf{0}_{V_{\alpha}} \in V$. 
Let us proceed to precise definitions. For each element $k \in K$, we denote by $k_{\alpha}$ the self-mapping of $V=\prod_{\alpha} V_{\alpha}$ that acts as the multiplication by $k$ of the $\alpha$ th component of each element $x \in V$ and does not alter any other components. A subset $S \subset V$ will be called an $\alpha$-fiber or simply a fiber if the projection $p_{\beta}(S)$ is a singleton for $\alpha \neq \beta$ and if $p_{\alpha}(S)=V_{\alpha}$. Thus, all but one components of elements of a fiber $S$ are fixed, whereas the $\alpha$ th component can take arbitrary values in $V_{\alpha}$.

A tensor, or a complete representation of a tensor, is an arbitrary subset $X \subset$ $V=\prod_{\alpha} V_{\alpha}$ with the following properties:

1) if $k_{\alpha}(x) \in X$ for some index $\alpha \in A$ and $k \in K$, then $k_{\beta}(x) \in X$ for all indices $\beta \in A$;

2) $\oplus(X \cap S) \in X$ for each fiber $S \subset V$;

3) if $y \in S, x \in X \cap S$, and $y \preccurlyeq x$, then $y \in X$ for each fiber $S \subset V$.

Needless to say, property 1) corresponds to Eq. (2), and properties 2) and 3) correspond to Eq. (3) with regard to the fact that $x \oplus y=x$ for $y \preccurlyeq x$. We shall assume that a complete representation $X$ always contains $\mathbf{0}_{V}=\left\{\mathbf{0}_{\alpha}\right\}$. Formally, this corresponds to the identity $\sup \varnothing=\mathbf{0}_{V}$.

We define the $\tau$-hull $X^{\tau}$ of an arbitrary subset $X \subset V=\prod_{\alpha} V_{\alpha}$ as the intersection of all tensors (that is, their complete representations) containing $X$. Two subsets $X, Y \subset V$ are said to be equivalent (we denote this equivalence relation by $\tau)$ if their $\tau$-hulls $X^{\tau}$ and $Y^{\tau}$ coincide.

An elementary analysis shows that the following assertion holds.

Proposition 3. The equivalence generated by (2) and (3) with regard to the fact that the addition is idempotent on the set of representations of the form (4) coincides with the equivalence $\tau$. Every representation of the form (1) is equivalent to a unique complete representation of the form (4). This equivalence is consistent with the structure of a semimodule over $K$ on $T$ and $T_{b}$.

It follows that the quotient semimodules $\widetilde{T}$ and $\widetilde{T}_{b}$ are well defined. One can readily see that the semimodule $\widetilde{T}_{b}$ is $b$-complete; in any case, this follows from Proposition 4 below. The quotient semimodule $\widetilde{T}_{b}$ will be denoted by $\bigotimes_{\alpha} V_{\alpha}$ and called the b-tensor (or simply tensor) product of the b-complete semimodules $V_{\alpha}$.

Recall that we identify a tensor with its complete representation, i.e., a subset $X X^{\tau} \subset V$.

We say that a tensor $X$ is a bounded or b-tensor if it is contained (as a set) in a tensor $\{x\}^{\tau}$, where $x \in V$. In this case we say that $X$ is bounded by the tensor $\{x\}^{\tau}$, i.e., the tensor with representation $\bigotimes_{\alpha} x_{\alpha}$, where $x=\left\{x_{\alpha}\right\}$.

Let $T_{b}(V)$ be the set of all $b$-tensors, ordered by inclusion. This order gives rise to an idempotent addition in $T_{b}(V)$. The sum of an arbitrary bounded family $\left\{X_{\alpha}\right\}$ of tensors coincides with the $\tau$-hull $\left\{\bigcup_{\alpha} X_{\alpha}\right\}^{\tau}$ of their unions. If a $b$-tensor $X$ is bounded by a tensor $\{x\}^{\tau}$ and a $b$-tensor $Y$ is bounded by a tensor $\{y\}^{\tau}$, then the tensor $X \oplus Y=\{X \cup Y\}^{\tau}$ is bounded by the tensor $\{x \oplus y\}^{\tau}$, where $x \oplus y \in V$. Clearly, the intersection of an arbitrary family of $b$-tensors is again a $b$-tensor, which coincides with the greatest lower bound of this family of tensors (with respect to the order defined by the set-theoretic inclusion of subsets of $V$ ). It follows that every subset $M \subset T_{b}(V)$ that is bounded above has the least upper bound

$$
\sup M=\inf \{X \mid Y \preccurlyeq X \text { for all } Y \in M\}=\bigcap_{X \supset Y \in M} X
$$


(e.g., see $[10])$. Thus $T_{b}(V)$ is a lattice and a $b$-complete idempotent semigroup.

One can multiply elements $X \in T_{b}(V)$ by elements $k \in K$ according to the formula $k \odot X=\left(k_{\alpha}(X)\right)^{\tau}$ for a given index $\alpha \in A$; by property 1 ) of the complete representation of a tensor, this multiplication is independent of the choice of $\alpha$.

Proposition 4. The idempotent semigroup $T_{b}(V)$ with the above-defined multiplication by elements of $K$ is a b-complete semimodule over $K$. This semimodule is isomorphic to the semimodule $\bigotimes_{\alpha} V_{\alpha}$.

Proof. Let us verify that $T_{b}(V)$ is a $b$-complete semimodule. We have already seen that $T_{b}(V)$ is a $b$-complete idempotent semigroup. Hence it suffices to verify the distributivity laws and the associativity of the multiplication by elements of $K$. One can readily verify that for each $b$-homomorphism $g: V \rightarrow V$ and each $b$-tensor $X \subset V$, the set $g^{-1}(X)$ is also a $b$-tensor. It follows that $g\left(X^{\tau}\right) \subset(g(X))^{\tau}$ and $\left(g\left(X^{\tau}\right)\right)^{\tau}=(g(X))^{\tau}$ for each subset $X \subset V$. By applying these relations to $b$ homomorphisms of the form $k_{\alpha}$, we find that $\left(k_{\alpha}(X)\right)^{\tau}=\left(k_{\alpha}\left(X^{\tau}\right)\right)^{\tau}$ for all $X \subset V$, $k \in K, \alpha \in A$, whence it follows that the multiplication by elements of $K$ is associative.

Let us verify the distributivity. For each subset $\mathcal{T} \subset T_{b}(V)$ that is bounded above, one has $\oplus \mathcal{T}=\left(\bigcup_{t \in \mathcal{T}} t\right)^{\tau}$. Consequently,

$k \odot(\oplus \mathcal{T})=k \odot\left(\bigcup_{t \in \mathcal{T}} t\right)^{\tau}=\left(k_{\alpha}\left(\left(\bigcup_{t \in \mathcal{T}} t\right)^{\tau}\right)\right)^{\tau}=\left(k_{\alpha}\left(\bigcup_{t \in \mathcal{T}} t\right)\right)^{\tau}=\left(\bigcup_{t \in \mathcal{T}} k_{\alpha}(t)\right)^{\tau}$

Since

$$
\left(\bigcup_{t \in \mathcal{T}} k_{\alpha}(t)\right)^{\tau}=\left(\bigcup_{t \in \mathcal{T}}\left(k_{\alpha}(t)\right)^{\tau}\right)^{\tau}=\bigoplus_{t \in \mathcal{T}} k \odot t=\oplus(k \odot \mathcal{T})
$$

we find that $k \odot(\oplus \mathcal{T})=\oplus(k \odot \mathcal{T})$, so that one of the distributivity laws follows. Here the small letter $t$ stands for a $b$-tensor, whereas above (and below) tensors are denoted by capital letters.

Let us now verify the second distributivity law $(\oplus Q) \odot X=\oplus(Q \odot X)$, where $Q$ is an arbitrary bounded subset in $K$ and $X$ is an arbitrary $b$-tensor. It suffices to verify that the intersections of these sets with the $\alpha$-fiber $S$ coincide for an arbitrary index $\alpha \in A$. However, by construction, $(\oplus Q)_{\alpha}(x)=\oplus(Q \odot x) \in\{k \odot x \mid k \in Q\}^{\tau}$ for any $x \in S$. Consequently, $(\oplus Q)_{\alpha}(x) \in\left(\bigcup_{k \in Q} k_{\alpha}(X)\right)^{\tau}$ for $x \in S \cap X$, whence it follows that $\left((\oplus Q)_{\alpha}(X)\right) \subset\left(\bigcup_{k \in Q} k_{\alpha}(X)\right)^{\tau}$ and hence $(\oplus Q) \odot X=\left((\oplus Q)_{\alpha}(X)\right)^{\tau} \subset$ $\left(\bigcup_{k \in Q} k_{\alpha}(X)\right)^{\tau}$. However, $\left(\bigcup_{k \in Q} k_{\alpha}(X)\right)^{\tau}=\left(\bigcup_{k \in Q}\left(k_{\alpha}(X)\right)^{\tau}\right)^{\tau}=\bigoplus_{k \in Q} k \odot X=$ $\oplus(Q \odot X)$. Thus $(\oplus Q) \odot X \preccurlyeq \oplus(Q \odot X)$. Since the opposite inequality is obvious, this completes the verification of the second distributivity law. Thus we have proved that $T_{b}(V)$ is a $b$-complete semimodule over $K$.

It remains to indicate (and verify) an isomorphism between $T_{b}(X)$ and $\bigoplus_{\alpha} V_{\alpha}$. Basically, now we can readily derive this from Proposition 3. We obtain a mapping $T_{b}(V) \rightarrow \bigoplus_{\alpha} V_{\alpha}$ by taking each $b$-tensor $X$ to its (complete) formal representation $\bigoplus_{x=\left\{x_{\alpha}\right\} \in X} \bigotimes_{\alpha} x_{\alpha}$ of the form (4) and by passing to the corresponding equivalence class in $T_{b}$. The inverse mapping takes an arbitrary representation of the form (1) or (4) to the complete representation of the form (4). The verification of the fact that these mappings are mutually inverse and the structures of semimodules over $K$ are consistent (coincide) is elementary. The proof of the proposition is complete.

Remark 3. Relation (2) and property 1) of the complete representation of a tensor show why we require the basic semiring $K$ to be commutative. 


\section{§4. Basic Results on b-Polylinear Mappings}

Let $\left\{V_{\alpha}\right\}_{\alpha \in A}$ be an arbitrary family of $b$-complete idempotent semimodules over a $b$-complete commutative semiring $K$, and let $W$ be an arbitrary $b$-complete semimodule over $K$. We set $V=\prod_{\alpha} V_{\alpha}$; thus, $V$ is the direct product of the semimodules $V_{\alpha}$; by $\bigotimes_{\alpha} V_{\alpha}$ we denote the $b$-tensor product of these semimodules.

A mapping $f: \prod_{\alpha} V_{\alpha} \rightarrow W$ is said to be $b$-polylinear if it is separately $b$-linear in every component $V_{\alpha}$; this means that for every given index $\beta \in A$ the mapping $x_{\beta} \mapsto f\left(\ldots, x_{\beta}, \ldots\right)$ for arbitrary fixed values of all other coordinates is a $b$-linear mapping $V_{\beta} \rightarrow W$.

We define a canonical mapping $\pi$ of the direct product $V=\prod_{\alpha} V_{\alpha}$ into the tensor product $T_{b}(V)=\bigotimes_{\alpha} V_{\alpha}$ by setting $\pi(x)=\{x\}^{\tau}$, i.e., $\pi\left(\left\{x_{\alpha}\right\}\right)=\bigotimes_{\alpha} x_{\alpha}$.

Remark 4. The range of the canonical mapping $\pi$ generates the semimodule $\bigotimes_{\alpha} V_{\alpha}$, i.e., each element of this semimodule is a linear combination (not necessarily finite) of elements of the form $\bigotimes_{\alpha} x_{\alpha}$; moreover, each element is a sum (not necessarily finite) of elements of the form $\bigotimes_{\alpha} x_{\alpha}$. In other words, the set $\pi\left(\prod_{\alpha} V_{\alpha}\right)$ is a system of generators of the semimodule $\bigotimes_{\alpha} V_{\alpha}$ and even of the corresponding idempotent semigroup.

Theorem 1. The canonical mapping $\left\{x_{\alpha}\right\} \mapsto \bigotimes_{\alpha} x_{\alpha}$ of the direct product $V=$ $\prod_{\alpha} V_{\alpha}$ of the semimodules $V_{\alpha}$ into the tensor product $T_{b}(V)=\bigotimes_{\alpha} V_{\alpha}$ of these semimodules is b-polylinear. For each b-polylinear mapping $f: \prod_{\alpha} V_{\alpha} \rightarrow W$ there exists a unique b-linear mapping $f_{\otimes}: \bigotimes_{\alpha} V_{\alpha} \rightarrow W$ such that $f=f_{\otimes} \pi$.

To prove this theorem, we need some auxiliary assertions. Just as above, we identify a tensor with its complete representation of the form (4), i.e., with the corresponding subset in $\prod_{\alpha} V_{\alpha}$. For each element $w$ of an arbitrary idempotent semigroup $W$, we denote by $\operatorname{Low}(w)$ the set $\{u \in W \mid u \preccurlyeq w\}$.

Lemma 1. Let $W$ be an arbitrary b-complete semimodule over $K$. For each $b$ polylinear mapping $f: \prod_{\alpha} V_{\alpha} \rightarrow W$, the complete preimage of each set of the form Low $(w)$, where $w \in W$, is a b-tensor.

Proof. Let $X=f^{-1}(\operatorname{Low}(w))$. If $x$ and $y$ are elements of some fiber, $x \in X$, and $y \preccurlyeq x$, then $f(y) \preccurlyeq f(x) \preccurlyeq w$, whence $y \in X$. If $S \subset X$ and $S$ is contained in a single fiber, then $f(\oplus S)=\oplus f(S) \preccurlyeq w$, whence $\oplus S \in X$. If $y=k_{\alpha}(x)$ and $k_{\beta}(x) \in X$ for some index $\beta$, then $f(y)=k \odot f(x)=f\left(\left(k_{\beta}(x)\right) \preccurlyeq w\right.$, whence $y \in X$. Thus conditions 1)-3) from the definition of a tensor are satisfied, which completes the proof of Lemma 1.

Lemma 2. For any b-polylinear mapping $f: \prod_{\alpha} V_{\alpha} \rightarrow W$ and any subset $X \subset$ $V=\prod_{\alpha} V_{\alpha}$, one has $\oplus f(X)=\oplus f\left(X^{\tau}\right)$.

Proof. It follows from Lemma 1 that the set $\{x \in V \mid f(x) \preccurlyeq w\}$ is a $b$-tensor for each $w \in W$. In particular, the set $\{x \in V \mid f(x) \preccurlyeq \oplus f(X)\}$ is a $b$-tensor containing $X$. It follows that it contains the set $X^{\tau}$, whence $\oplus f(X) \succcurlyeq \oplus f\left(X^{\tau}\right)$. Since the opposite inequality is obvious, the proof of Lemma 2 is complete.

Proof of Theorem 1. Let us verify that the canonical mapping is $b$-polylinear. To this end, let us verify that $\pi(\oplus X)=\oplus \pi(X)$ for each subset $X \subset V=\prod_{\alpha} V_{\alpha}$ lying in an $\alpha$-fiber $S \subset V$. Since $\oplus X \in X^{\tau}$, we have the inclusion $\pi(\oplus X) \subset X^{\tau}$. On the other hand, if $x \in X$, then $x \in S, \oplus X \in S$, and $x \preccurlyeq \oplus X$, whence 
$x \in(\oplus X)^{\tau}$. Consequently, $X \subset(\oplus X)^{\tau}$, whence $X^{\tau} \subset(\oplus X)^{\tau}=\pi(\oplus X)$, so that $\oplus \pi(X)=X^{\tau}=\pi(\oplus X)$, as desired. Since the relation $\pi\left(k_{\alpha}(x)\right)=k \odot \pi(x)$, where $x \in V, k \in K$, readily follows from the definition of the multiplication of a $b$-tensor by an element $k \in K$, we have proved that the canonical mapping $\pi$ is $b$-polylinear.

It remains to verify the existence and uniqueness of the mapping $f_{\otimes}$. For each $b$-tensor $t$, we set $f_{\otimes}(t)=\oplus f(t)$, where $f(t)$ is the image of the set $t$ (the complete representation of the tensor $t$ ) under the mapping $f$. It follows from Lemma 2 that this is well-defined and that $f_{\oplus}(\pi(x))=f(x)$.

For each subset $T$ of the tensor product $\otimes V_{\alpha}$, one has

$$
f_{\otimes}(\oplus T)=f_{\otimes}\left(\left(\bigcup_{t \in T} t\right)^{\tau}\right)=\oplus f\left(\bigcup_{t \in T} t\right)=\bigoplus_{t \in T}(\oplus f(t))=\oplus f_{\otimes}(T)
$$

so that $f_{\otimes}$ is a $b$-homomorphism of the corresponding idempotent semigroups.

One can also readily prove that the mapping $f_{\otimes}$ is homogeneous. Indeed,

$$
f_{\otimes}(k \odot \pi(x))=f_{\otimes}\left(\pi\left(k_{\alpha}(x)\right)\right)=f\left(k_{\alpha}(x)\right)=k \odot f(x)=k \odot f_{\otimes}(\pi(x)) .
$$

Thus $f_{\otimes}$ is homogeneous on $\pi(V)$. Since $f_{\otimes}$ is a $b$-homomorphism and the range $\pi(V)$ of the canonical mapping generates $\bigotimes_{\alpha} V_{\alpha}$ (see Remark 4), this completes the proof of the homogeneity of the mapping $f_{\otimes}$. The uniqueness of this mapping also follows from Remark 4. The proof of Theorem 1 is complete.

Theorem 2. Suppose that $V=\prod_{\alpha} V_{\alpha}, W$ is a b-complete semimodule over $K$, and there is a b-polylinear mapping $\varepsilon: V \rightarrow W$ whose range generates the semimodule $W$, so that each element of $W$ is a linear combination of elements of the form $\varepsilon(x)$. Suppose that for each b-polylinear mapping $f: V \rightarrow U$, where $U$ is an arbitrary $b$-complete semimodule over $K$, there exists a b-linear mapping $F: W \rightarrow U$ such that $F \varepsilon=f$. Then there exists an isomorphism $\delta: W \rightarrow \bigotimes_{\alpha} V_{\alpha}$ of the semimodule $W$ onto the b-tensor product $\bigotimes_{\alpha} V_{\alpha}$ such that $\delta \varepsilon=\pi$.

Proof. Let $\delta: W \rightarrow \bigotimes_{\alpha} V_{\alpha}$ be a $b$-linear mapping such that $\delta \varepsilon=\pi$. This mapping exists by the assumption of Theorem 2 applied to the $b$-polylinear mapping $f=\pi$. On the other hand, by applying Theorem 1 to the $b$-polylinear mapping $\varepsilon$, we can construct a $b$-linear mapping $\varepsilon_{\otimes}: \bigotimes_{\alpha} V_{\alpha} \rightarrow W$ such that $\varepsilon_{\otimes} \pi=\varepsilon$. Since $\delta \varepsilon_{\otimes} \pi=\delta \varepsilon=\pi$, it follows that the $b$-linear mapping $\delta \varepsilon_{\otimes}$ leaves all elements of $\pi(V)$ in their places; now we see that this mapping coincides with the identity mapping, since $\pi(V)$ generates $\bigotimes_{\alpha} V_{\alpha}$. Likewise, one can check that $\varepsilon_{\otimes} \delta$ is also the identity mapping. Thus, the $b$-linear mappings $\varepsilon_{\otimes}$ and $\delta$ are the inverses of each other and are isomorphisms. Thus Theorem 2 follows from Theorem 1.

Remark 5. Theorems 1 and 2 show that in the category of $b$-complete semimodules (with $b$-linear mappings as morphisms) we have constructed a natural tensor product. This tensor product is also a natural tensor product in the full subcategory of $a$-complete semimodules, since one can readily see that the $b$-tensor product of an arbitrary family of $a$-complete semimodules (over a given idempotent semiring) is an $a$-complete semimodule.

By using similar constructions, one can readily construct a tensor product (with finitely many factors) in the category of arbitrary idempotent semimodules over a given commutative idempotent semiring (with linear mappings as morphisms). 


\section{$\S 5$. Tensor Products of $b$-Linear Mappings}

Let $\left\{V_{\alpha}\right\}_{\alpha \in A}$ and $\left\{W_{\alpha}\right\}_{\alpha \in A}$ be families of $b$-complete semimodules over a given $b$-complete commutative semiring $K$. Suppose that for each index $\alpha \in A$ there is a $b$-linear mapping $f_{\alpha}: V_{\alpha} \rightarrow W_{\alpha}$. By $f$ we denote the direct product $\prod_{\alpha} f_{\alpha}$ of these mappings, i.e., the mapping $f: \prod_{\alpha} V_{\alpha} \rightarrow \prod_{\alpha} W_{\alpha}$ such that $f\left(\left\{x_{\alpha}\right\}\right)=\left\{f\left(x_{\alpha}\right)\right\}$. One can readily see that this mapping extends to be a $b$-polylinear mapping

$$
\prod_{\alpha} V_{\alpha} \rightarrow \bigotimes_{\alpha} W_{\alpha}
$$

It follows from Theorem 1 that the mapping (5) extends to be a $b$-linear mapping

$$
\bigotimes V_{\alpha} \rightarrow \bigotimes_{\alpha} W_{\alpha}
$$

The mapping (6) is called the b-tensor (or simply tensor) product of the b-linear mappings $f_{\alpha}$ and is denoted by $\bigotimes_{\alpha} f_{\alpha}$.

\section{§6. The Tensor Algebra}

Let $U, V$ and $W$ be $b$-complete semimodules over a $b$-complete commutative idempotent semiring $K$. We use the symbol $\dot{+}$ to denote the direct sum of two semimodules and the symbol $\sum_{\alpha} V_{\alpha}$ to denote the direct sum of an arbitrary family of $a$-complete semimodules. In these cases the direct sum coincides with the direct product (see Remark 2).

Theorem 3. The following b-complete semimodules are isomorphic:

1) $U \otimes V$ and $V \otimes U$;

2) $(U \dot{+} V) \otimes W$ and $U \otimes W \dot{+} V \otimes W$

3) $(U \otimes V) \otimes W$ and $U \otimes(V \otimes W)$;

4) $\left(\sum_{\alpha} V_{\alpha}\right) \otimes W$ and $\sum_{\alpha} V_{\alpha} \otimes W$,

where $\left\{V_{\alpha}\right\}_{\alpha \in A}$ is an arbitrary family of a-complete semimodules over $K$.

Corollary. The set of isomorphism classes of b-complete semimodules (over a given b-complete commutative idempotent semiring) is a commutative associative semiring with respect to the operations of direct sum and b-tensor product.

Remark 6. This semiring of semimodules is not idempotent.

Proof of Theorem 3. Assertion 1) is trivial and readily follows from the definition of the $b$-tensor product. Assertion 3 ) follows from the fact that the direct products $U \times(V \times W)$ and $(U \times V) \times W$ are isomorphic, and moreover, the set of $b$-tensors (i.e., of their complete representations of the form (4)) is the same in both cases and coincides with the set of $b$-tensors in $U \times V \times W$, i.e., with $U \otimes V \otimes W$, as desired.

Let us prove assertions 2) and 4). Suppose that the direct sum $\sum_{\alpha} V_{\alpha}=V$ of the family of semimodules $V_{\alpha}$ exists and coincides with the direct product $\prod_{\alpha} V_{\alpha}$ (see Remark 2); for assertion 2) this family is finite (or even consists of two semimodules). Let $i_{\alpha}: V_{\alpha} \rightarrow V$ and $p_{\alpha}: V \rightarrow V_{\alpha}$ be the corresponding canonical embeddings and projections, and let $i: \sum_{\alpha}\left(V_{\alpha} \otimes W\right) \rightarrow V \otimes W$ be the direct sum of the mappings $i_{\alpha} \otimes I_{W}$, where $I_{W}$ is the identity operator in $W$ (the tensor product of $b$-linear 
mappings was defined in $\S 5)$. Thus the restriction of the mappings $i$ to $V_{\alpha} \otimes W$ coincides with $i_{\alpha} \otimes I_{W}$. Likewise, let $p: V \otimes W \rightarrow \sum_{\alpha}\left(V_{\alpha} \otimes W\right)=\prod_{\alpha}\left(V_{\alpha} \otimes W\right)$ be the direct product of the mappings $p_{\alpha} \otimes I_{W}$. A routine verification shows that $i$ and $p$ are mutually inverse isomorphisms.

\section{§7. Tensor Products of Semimodules of Bounded Functions}

Let $K$ be a $b$-complete commutative idempotent semiring and $X$ an arbitrary set. By $\mathcal{B}(X, K)$ we denote the set of bounded functions on $X$ ranging in $K$, i.e., mappings $X \rightarrow K$ with bounded range. If $f, g \in \mathcal{B}(X, K)$, then the pointwise addition and multiplication

$$
(f \oplus g)(x)=f(x) \oplus g(x), \quad(f \odot g)(x)=f(x) \odot g(x),
$$

where $x$ is an arbitrary element of $X$, define a structure of a $b$-complete semimodule over $K$ on $\mathcal{B}(X, K)$ (e.g., see $[17,18,5,12])$.

Proposition 5. For any sets $X$ and $Y$, the $b$-complete semimodules $\mathcal{B}(X \times Y, K)$ and $\mathcal{B}(X, K) \otimes \mathcal{B}(Y, K)$ are isomorphic.

Proof. Let $\delta_{x} \in \mathcal{B}(X, K)$ be the function equal to $\mathbf{1}$ at the point $x \in X$ and $\mathbf{0}$ at all other points. One can readily verify that the mapping

$$
(f, g) \mapsto \oplus\left\{f(x) \odot f(y) \odot \delta_{(x, y)}\right\}
$$

is a $b$-linear mapping $\mathcal{B}(X, K) \times \mathcal{B}(Y, K) \rightarrow \mathcal{B}(X \times Y, K)$; hence it extends to be a $b$-linear mapping

$$
i: \mathcal{B}(X, K) \otimes \mathcal{B}(Y, K) \rightarrow \mathcal{B}(X \times Y, K) .
$$

We define a $b$-linear mapping

$$
j: \mathcal{B}(X \times Y, K) \rightarrow \mathcal{B}(X, K) \otimes \mathcal{B}(Y, K)
$$

by setting $j(f)=\oplus\left\{f(x, y) \odot \delta_{x} \otimes \delta_{y}\right\}$; this is obviously well defined. One can readily see that the mappings $(7)$ and (8) are the inverses of each other and specify the desired homomorphism. It suffices to verify this on a system of generators. For an element $(x, y) \in X \times Y$, one readily has $i\left(j\left(\delta_{(x, y)}\right)\right)=i\left(\delta_{x} \otimes \delta_{y}\right)=\delta_{(x, y)}$, so that $i j$ is the identity mapping on $\mathcal{B}(X \times Y, K)$. On the other hand, elements of the form $\delta_{x} \otimes \delta_{y}$ obviously generate $\mathcal{B}(X, K) \otimes \mathcal{B}(Y, K)$, and $j\left(i\left(\delta_{x} \otimes \delta_{y}\right)\right)=j\left(\delta_{(x, y)}\right)=$ $\delta_{x} \otimes \delta_{y}$; it follows that $j i$ is the identity mapping. The proof of the proposition is complete.

\section{$\S 8$. Nuclear Operators on $b$-Complete Semimodules}

Let $V$ and $W$ be $b$-complete semimodules over a $b$-complete commutative idempotent semiring $K$. By $W^{*}$ we denote the semimodule of all $b$-linear mappings $W \rightarrow K$ (b-linear functionals). The set of all $b$-linear mappings $W \rightarrow V$ is denoted by $\operatorname{Hom}_{b}(W, V)$. This set bears a natural structure of a $b$-complete semimodule over $K$. Thus $W^{*}=\operatorname{Hom}_{b}(W, K)$.

For $v \in V$ and $w^{*} \in W^{*}$ we define an operator (i.e., a $b$-linear mapping) $p_{w^{*}, v}: W \rightarrow V$ by the formula

$$
p_{w^{*}, v}: x \mapsto w^{*}(x) \odot v .
$$

Operators of the form (9) will be called operators of rank 1 (or one-dimensional operators). 
Proposition 6. There exists a unique b-linear mapping $p: W^{*} \otimes V \rightarrow \operatorname{Hom}_{b}(W, V)$ such that $p\left(w^{*} \otimes v\right)=p_{w^{*}, v}$. The range of the operator $p$ is the subsemimodule of $\operatorname{Hom}_{b}(W, V)$ generated by the set of all operators of the form (9), i.e., by operators of rank 1.

To prove this, it suffices to notice that the mapping $W^{*} \times V \rightarrow \operatorname{Hom}_{b}(W, V)$ taking each pair $\left(w^{*}, v\right)$ to the operator $p_{w^{*}, v}$ is $b$-bilinear and its range consists of all operators of rank 1; then we can apply Theorem 1 . The mapping $p$ described in Proposition 6 will be called canonical.

By analogy with [1], we refer to a $b$-linear mapping $n: W \rightarrow V$ as a $b$-nuclear operator (or a nuclear mapping) if $n$ lies in the range of the canonical mapping $p$. It follows from Proposition 6 that a nuclear mapping $W \rightarrow V$ can be represented as a sum of operators of rank 1. It readily follows from this proposition and from our definitions that the following assertion holds.

Corollary. If the semimodules $V$ and $W$ are a-complete, then the range of the canonical mapping $p$ is an a-complete subsemimodule of $\operatorname{Hom}_{b}(W, V)$; a b-linear mapping $W \rightarrow V$ is nuclear if and only if it can be represented as a sum (possibly, infinite) of operators of rank 1.

The authors intend to carry out a detailed study of $b$-nuclear operators in connection with idempotent analogs of kernel theorems (in the spirit of Schwartz and Grothendieck) in one of the subsequent papers. Here we only consider the following example.

Example (see $[17,18])$. Let $W=\mathcal{B}(X, K)$ and $V=\mathcal{B}(Y, K)$ (as described in $\S 7$ ). By the kernel theorem [18], every b-linear operator $f: W \rightarrow V$ has the form

$$
f: \varphi(x) \mapsto \widetilde{\varphi}(y)=\int_{X}^{\oplus} K_{f}(x, y) \odot \varphi(x) d x=\sup _{x \in X}\left(K_{f}(x, y) \odot \varphi(x)\right),
$$

where $\varphi \in W, K_{f}(x, y) \in \mathcal{B}(X \times Y, K)$ and the "idempotent integral" $\int_{X}^{\oplus} \psi(x) d x$ (e.g., see $[2,4-8]$ ) is defined as $\sup _{x \in X} \psi(x)$ for each function $\psi \in \mathcal{B}(X, K)$. We define a $b$-linear functional $K_{f, y}$ on $W$ by setting

$$
K_{f, y}(\varphi)=\int_{X}^{\oplus} K_{f}(x, y) \varphi(x) d x .
$$

The integral representation (10) can be rewritten in the form $f(\varphi)=\bigoplus_{y \in Y} K_{f, y}(\varphi) \odot$ $\delta_{y}$. It follows from the kernel theorem that each $b$-linear operator $f: \mathcal{B}(X, K) \rightarrow$ $\mathcal{B}(Y, K)$ is $b$-nuclear. It follows from the theorem on the structure of a $b$-linear functional [18] that each $b$-nuclear operator $f: W \rightarrow V$ can be specified by an "integral" kernel $K_{f}(x, y)$. Thus, the existence of an integral representation of an operator is provided by the fact that the operator is nuclear and by the existence of integral representations of $b$-linear functionals. This scheme can be generalized to a wide class of $b$-complete semimodules.

Remark 7. Note that $W^{*}=\left(\widehat{W}_{b}\right)^{*}$, where $\widehat{W}_{b}$ is the $b$-completion of the semimodule $W$ (the procedure of $b$-completion for semimodules is discussed, for example, in [12]). Hence the requirement that the semimodule $W$ be $b$-complete is in fact not very important. In general, if semimodules $V$ and $W$ over $K$ admit $b$-completions 
$\widehat{V}_{b}$ and $\widehat{W}_{b}$ over $\widehat{K}_{b}$, then the "completed" $b$-tensor product $V \widehat{\otimes} W$ can be defined as $\widehat{V}_{b} \otimes \widehat{W}_{b}$.

Remark 8. For the case of a-complete idempotent semimodules (and tensors products of finite families of semimodules of this type), some results of the present paper are also contained (in other terms and under a different approach) in [19], where a rather general categorical approach to tensor products, suggested in [20] (see also [21]), was studied. Note that the approach of [20] is not always convenient for applications to analysis in the spirit of A. Grothendieck [1]. For example, for the category of locally convex (or Banach) spaces this approach provides only one of all possible (and important in analysis) topological tensor products in the sense of [1]. In forthcoming papers, we shall consider various versions of idempotent analogs of topological tensor products.

\section{Acknowledgements}

This research was supported by INTAS and the Russian Foundation for Basic Research under joint grant No. 95-91.

\section{REFERENCES}

1. A. Grothendieck, Produits tensoriels topologiques et espaces nucléairs, Mem. Amer. Math. Soc., 16, Providence (R.I.) (1955).

2. V. P. Maslov, Asymptotic Methods for Pseudodifferential Equations [in Russian], Nauka, Moscow (1987).

3. V. P. Maslov, "A new superposition principle for optimization problems," Uspekhi Mat. Nauk, 42, No. 3, 39-48 (1987) [in Russian; English Transl. in: Russian Math. Surveys 42 (1987), no. $3,43-54$.

4. V. P. Maslov, Méthodes opératorielles, Mir, Moscow (1987).

5. V. P. Maslov and S. N. Samborskiŭ, editors, Idempotent Analysis, Vol. 13 in Adv. Soviet Math, Amer. Math. Soc., Providence (R.I.) (1992).

6. V. P. Maslov and V. N. Kolokoltsov, Idempotent Analysis and Its Applications in Optimal Control Theory [in Russian], Nauka, Moscow (1994).

7. V. N. Kolokoltsov and V. P. Maslov, Idempotent Analysis and Applications, Kluwer Acad. Publ., Dordrecht (1997).

8. G. L. Litvinov and V. P. Maslov, Correspondence Principle for Idempotent Calculus and Some Computer Applications, Preprint IHES/M/95/33, Institut des Hautes Etudes Scientifiques, Bures-sur-Yvette (1995) (see also [9, pp. 420-443], and http://arXiv.org/abs/math.GM/0101021)

9. Idempotency (J. Gunawardena, editor), Publ. of the Newton Institute, Cambridge Univ. Press, Cambridge (1998).

10. G. Birkhoff, Lattice Theory, 3d edition, American Mathematical Society Colloquium Publications, Vol. XXV, American Mathematical Society, Providence, R.I. (1967).

11. G. L. Litvinov, V. P. Maslov, and G. B. Shpiz, "Linear functionals on idempotent spaces. An algebraic approach," Dokl. Ross. Akad. Nauk, 363, No. 3, 298-300 (1998); English transl. in: Doklady Mathematics, 58, No. 3, 389-391 (1998) (see alsohttp://arXiv.org/abs/math.FA/0012268)

12. G. L. Litvinov, V. P. Maslov, and G. B. Shpiz, Idempotent Functional Analysis. I. An Algebraic Approach, Preprint, International Sophus Lie Center, Moscow ,1998 (see also http://arXiv.org/abs/math.FA/0009128).

13. F. L. Bacelli, G. Cohen, G. J. Olsder, and J. -P. Quadrat, Synchronization and Linearity: an Algebra for Discrete Event Systems, Wiley, New York (1992).

14. M. Gondran and M. Minoux, Graphes et algorithms, Clarendon Paperbacks, Oxford Univ. Press, Oxford (1979).

15. H. Schäfer, Topological Vector Spaces, Graduate Texts in Mathematics, Vol. 3, Springer-Verlag, New York-Berlin (1971).

16. S. I. Gelfand and Yu. I. Manin, Methods in Homological Algebra. Vol. 1. Introduction to Cohomology Theory and Derived Categories [in Russian], Nauka, Moscow (1988).

17. P. I. Dudnikov and S. N. Samborskii, "Endomorphisms of semimodules over semirings with idempotent operations," (Kiev: Institute for Mathematics, Akad. Nauk Ukr. SSR, 1987), Izv. Akad. Nauk SSSR Ser. Mat. [Math. USSR-Izv.], 55, No. 1, 91-105 (1991). 
18. M. A. Shubin, Algebraic Remarks on Idempotent Semirings and a Kernel Theorem in Spaces of Bounded Functions [in Russian], Institute for New Technology, Moscow (1990). English transl. in [5], 151-166.

19. A. Joyal and M. Tierney, "An extension of the Galois theory of Grothendieck," Mem. Amer. Math. Soc., 51, No. 309 (1984).

20. B. Banaschewski and E. Nelson, "Tensor products and bimorphisms," Canad. Math. Bull., 19, No. 4, 385-402 (1976).

21. D. Pumplün, "Das Tensorprodukt als universelles Problem," Math. Ann., 171, 247-262 (1967).

International Sophus Lie Center (G. L. Litvinov and G. B. Shpiz)

litvinov@islc.msk.su

Moscow State University (V. P. Maslov)

maslov@ipmnet.ru 